\title{
MicroRNA-21, 204 and 125b Play Potential Roles in Tumorigenesis of Melanoma
}

\author{
Chengtan Li ${ }^{*}$, Xiayu Wang1*, Ya'ni Chen ${ }^{1}$, Xiaohua Tan1, Wen Li², Sheng Yan', Weibin Cai', \\ Xianrong Xu' ${ }^{1}$, Liangwen Xu1, Lei Yang1\#, Yutao Yan1\# \\ ${ }^{1}$ School of Medicine, Hangzhou Normal University, Hangzhou, China \\ ${ }^{2}$ IHRC Inc., Atlanta, GA, USA \\ Email:"yanglei62@yahoo.com, "yutaoyan@yahoo.com
}

Received 3 November 2015; accepted 5 December 2015; published 10 December 2015

Copyright (C) 2015 by authors and Scientific Research Publishing Inc.

This work is licensed under the Creative Commons Attribution International License (CC BY). http://creativecommons.org/licenses/by/4.0/

(c) (i) Open Access

\section{Abstract}

Aim: The high mortality rate of melanoma is due to partially the lack of good diagnostic markers and treatment strategies. Over the past several years, several microRNA (miRNA) profiling studies have been performed on melanoma tissues, but with extremely inconsistency, the diagnostic value of miRNA candidates in melanoma remains under debate. Thus, this study aims to systematically evaluate the consistency of miRNAs tissue in multiple independent studies in melanoma. Method: Eligible studies were screened and selected from the PubMed, EMBASE, and Web of Science. A systematic analysis of published miRNA expression studies that compared the miRNA expression profiles between melanoma tissues and normal skin tissue was conducted. A vote-counting strategy was followed with the collection of information. Real time PCRs were employed to validate miRNA candidates with high consistency. Targets of consistent miRNAs were predicted by online programs (like miRTarBase, microRNA.org and TargetScanHuman 6.2). Enrichment analyses for gene ontology (GO) terms and Kyoto encyclopedia of genes and genomes (KEGG) pathways were carried out with Database for Annotation, Visualization, and Integrated Discovery (DAVID). Results: A total of 303 differentially expressed miRNAs were reported in the 10 miRNA-profiling studies during comparison of melanoma tissues with normal tissues; 132 were up-regulated in melanoma, and 171 were down-regulated. However, in the group of consistently reported miRNAs (cutoff $>3$ times), only moderate numbers of consistent and differentially expressed miRNAs were selected. miRNA-21 was found increased in 5 different studies, miRNA-146b, miRNA-17 and miRNA-18a were reported up-regulated in 4 profiling studies. Meanwhile, miRNA-204 and miRNA125b were found down-regulated in 5 studies, miRNA-141, miRNA-149, miRNA-224, miRNA-200b, miRNA-200c were consistently decreased in just 4 out of 10 profiling studies in total. The directions of differential expression of these miRNA candidates were confirmed by real time PCRs. Enrichment analyses demonstrated that programmed cell death and transcription regulation

\footnotetext{
${ }^{*}$ Chengtan Li and Xiayu Wang contribute to the manuscript equally.

${ }^{*}$ Corresponding author.
} 
played very important roles in the involvement of miRNAs in tumorigenesis of melanoma. Conclusion: This systematic study of melanoma miRNA profiling studies would provide rich information on miRNAs with potential role as the biomarkers and therapeutic agents with high consistency in melanoma.

\section{Keywords}

\section{Melanoma, miRNA, Systematic Analysis, Programmed Cell Death, Transcription}

\section{Introduction}

Cutaneous melanoma, as currently the sixth most common cancer in white men and women in the United States, is the most aggressive form of skin cancer characterized by poor prognosis [1], with the median no more than 1 year of survival time and is less than 5\% of 5-year survival rate [2] [3]. The incidence and mortality of melanoma continues to increase faster than any other cancer worldwide and there is no curative therapy for advanced stages of melanoma [4] [5]; as an example, almost 70 thousand new cases of melanoma and around 9 thousand deaths were predicted in 2009 [6]. Unfortunately, current treatment options are limited for metastatic melanoma. Therefore, new indicators of prognosis and therapeutic targets are pressing required. Genetic factors and exposure to ultraviolet radiation are risk factors for melanoma pathogenesis [7].

Since discovered in 1993, microRNAs (miRNAs), as small, evolutionarily conserved, single-stranded and non-coding RNA molecules, have been found to be important to regulate the expression of up to $30 \%$ encoding genes by binding to specific mRNA targets and promoting their degradation and/or translational inhibition [8], implying their important roles in physiological function and pathological condition. Growing evidence suggests that miRNAs, besides for a variety of essential cellular processes including development, cell differentiation, inflammation, proliferation, and apoptosis [9], play very important roles in initiating tumorigenesis [10], functioning as tumor suppressors or oncogenes (oncomir), evidenced by the miRNA deregulation in various cancer types like lung cancer [11]. Thereafter, miRNAs are implied as biomarkers and intervention targets against various forms of cancer. Further, miRNAs can be a superior method for cancer subtype classification and prognostication [12] [13]. An important feature of miRNAs is their remarkable stability; they can be well preserved in tissue samples even after years of formalin-fixation and paraffin embedding, and can be efficiently extracted from such specimens and variety of cell lines [14]. Several studies have been conducted to search for biomarkers or therapeutic agents by identifying the differential expression of miRNAs between melanoma tissue samples and corresponding non-tumor skin tissue from the same patient [15]-[25]. These studies generated hundreds of differentially expressed miRNAs, however, many of these are likely to be false positives and finally only a small number of them may have clinical significance and act as diagnostic and prognostic biomarkers. Further, different profiling studies showed quite inconsistency in the identified differentially expressed miRNAs. Third, in miRNA-based therapeutics, miRNAs are tissue specific; their role depends on cellular context. For instance, overexpression of miR-143 in pancreatic xenografts reduces tumor growth, and anti-miR-143 is required to suppress skin tumor formation [26]. It is therefore crucial to identify skin tissue specific and consistently differentially expressed miRNAs to investigate the potential as biomarkers or therapeutic agents. Thus, a logical approach to distinguish important miRNAs from a large number of candidate miRNA lists is to search for the intersection of miRNAs identified in multiple independent studies [27]. Although this kind of methods has become increasingly popular, no published study has identified the intersections of melanoma related miRNAs based on a large number of miRNA expression profiling studies thus far. Abnormal activation and inactivation of oncogenes and tumor suppressor genes are important factors leading to malignancy (including melanoma). Clarifying the molecular mechanisms of melanoma could provide a basis for melanoma risk assessment, early diagnosis, effective treatment and intervention.

We conducted this systematic analysis to identify the most important differentially expressed miRNAs that had been consistently reported in a series of independent miRNA expression profiling studies in melanoma patients. Moreover, we further validated some of the miRNAs that were most up- or down-regulated using realtime PCR in 6 pairs of melanoma and matched adjacent non-tumor tissue samples. 


\section{Materials and Methods}

\subsection{Search Strategy}

Potential studies published in English were collected from PubMed, EMBASE, Web of Science published from June, 2006 to Feb 2015 (last accessed on April 15, 2015) using the following medical subject headings terms: “miRNA” OR “microRNA” OR “miR”, “skin cancer” OR “melanoma”, "profiling” OR “microarray”.

\subsection{Inclusion Criteria of the Literature}

For a study to be included in this systematic review, several criteria had to be met: 1) studies had to be miRNA profiling studies in melanoma patients; 2) studies had to use melanoma tissues and their corresponding adjacent non-tumor tissues for comparison; 3) miRNA microarray and sequencing methods were comprised; they reported cut-off criteria of differentially expressed miRNAs, and 4) they reported the validation method set. Therefore, miRNA profiling studies that used serum samples of melanoma patients or skin relevant cell lines, those that compared melanoma biopsies from tumors with different stages of disease, or those using different miRNA techniques were excluded. Review articles were also excluded.

\subsection{Data Extraction}

Two investigators (X.W. and Y.C.) independently evaluated and extracted the data using standard protocols, and all discrepancies were resolved by the corresponding investigator (Y.Y.). From the full text and corresponding supplementary information, the following eligibility items were collected and recorded for each study: author, journal and year of publication, location of study, methods and characteristics of animal modeling, platform of miRNA expression profiling, author defined cut-off criteria of statistically significant differentially expressed miRNAs.

\subsection{Ranking}

Each included profiling/microarray study [15]-[25] provided a list of differentially expressed miRNAs related to melanoma. For example, Poliseno et al. [24] carried out comprehensive analysis of melanoma-related miRNAs based on a large number of miRNA profiling studies. Griffith et al. devised a method to rank potential molecular biomarkers for comparison groups [28], which had been used for miRNA profiling like meta-analysis studies. Briefly, miRNAs were ranked to the criteria in the following order of importance: (i) the miRNA was consistently reported as differentially expressed in a consistent direction of change; (ii) the frequency of the miRNAs was reported in the microarray studies; (iii) the total sample size for each consistent reported miRNAs.

\subsection{Validation of the miRNAs Using Real Time PCR}

To validate the profiling results, 6 fresh melanoma tissues and their paired non-tumor skin tissues were obtained from the Second Hospital of Hangzhou City, affiliated to Hangzhou Normal University School of Medicine. Total RNA was extracted from 6 pairs of matched human melanoma specimens (including cancer and adjacent noncancerous tissues) using TRIzol reagent (Life technology, Casbad, CA, USA) according to the manufacturer's instructions. The differentially expressed amount of the miRNAs was validated in triplicate by real time PCR. Briefly, Reverse transcription from 3 ug RNA was done using SuperScript III First-Strand Synthesis System (Life technology, Casbad, CA, USA) according to the manufacturer's protocol. Real-time PCR was performed using iQ SYBR Green Supermix kit (Bio-Rad, Hercules, CA) with the iCycler sequence detection system (Bio-Rad) with specific primers (Table 1). Small nucleolar RNA U6 was used as an internal standard for normalization. The cycle threshold $\left(\mathrm{C}_{\mathrm{T}}\right)$ value was calculated. The $2^{-\Delta \mathrm{CT}}\left(\Delta \mathrm{C}_{\mathrm{T}}=\mathrm{C}_{\mathrm{TmiR}}-\mathrm{C}_{\mathrm{TU} 6 \mathrm{RNA}}\right)$ method was used to quantify the relative amount of miRNAs.

\subsection{Identify the Experimentally Validated microRNA Target Genes}

miRTarBase (mirtarbase.mbc.nctu.edu.tw), microRNA.org and TargetScanHuman 6.2 were used to explore all the target genes of miRNAs, and highlighted the genes that are experimentally validated by luciferase reporter assay, western blot, or microarray experiments with over expression or knock- down of miRNAs. 
Table 1. Primers used for real time PCR.

\begin{tabular}{|c|c|c|}
\hline Primers & & Sequence \\
\hline miR-204 & For 5’ GGCTACAGTCTTTCTTCATG 3' & Rev 5' GCCAGTGATGACAATTGAACGTC 3' \\
\hline miR-141 & For 5' CCATCTTCCAGTACAGTGTTGG 3' & Rev 5' GCCATCTTTACCAGACAGTGTTAG 3' \\
\hline miR-125b & For 5' TGCGCTCCTCTCAGTCCCTGAG 3' & Rev 5’ AGCACGACTCGCAGCTCCCA 3' \\
\hline miR-149 & For 5’ GAGCTCTGGCTCCGTGTCT 3' & Rev 5’ TCCAGCTGCCCCAGCACAG 3’ \\
\hline $\operatorname{miR}-224$ & For 5' GCTTTCAAGTCACTAGTGGTTC 3' & Rev 5' GCTTTGTAGTCACTAGGGCACCA 3' \\
\hline miR-200c & For 5' CTCGTCTTACCCAGCAGTG 3' & Rev 5' CCTCCATCATTACCCGGCAG 3' \\
\hline miR-200b & For 5' CCAGCTCGGGCAGCCGTGG 3' & Rev 5’ CGTGCAGGGCTCCGCCGTCATC 3’ \\
\hline miR-17 & For 5’ GTCAGAATAATGTCAAAGT 3' & Rev 5' GTCACCATAATGCTACAAGTG 3' \\
\hline miR-21 & For 5’ TGTCGGGTAGCTTATCAGAC 3' & Rev 5' TGTCAGACAGCCCATCGACTG 3' \\
\hline miR-146b & For 5' CCTGGCACTGAGAACTGAA 3' & Rev 5' CACCAGAACTGAGTCCACAGGGC 3' \\
\hline miR-18a & For 5' GTTCTAAGGTGCATCTAGTG 3' & Rev 5’ GCCAGAAGGAGCACTTAGGGC 3' \\
\hline U6 & For 5' GCGCGTCGTGAAGCGTTC 3' & Rev 5' GTGCAGGGTCCGAGGT 3' \\
\hline
\end{tabular}

\subsection{Enrichment Analysis}

Enrichment analyses for gene ontology (GO) terms and Kyoto encyclopedia of genes and genomes (KEGG) pathways were carried out with Database for Annotation, Visualization, and Integrated Discovery (DAVID) [29]. DAVID is a web-accessible program that integrates functional genomics annotations with intuitive graphical summaries. List of gene or protein identifiers are rapidly annotated and summarized according to shared categorical data for Gene Ontology, protein domain, and biochemical pathway. For this, we listed all the target genes of all the differentially expressed miRNAs, including up-regulated and down-regulated miRNAs. The top 17 or $18 \mathrm{GO}$ terms and KEGG pathways showing association with target genes were listed with GO terms, KEGG pathway, number of genes in the GO term, number of genes in the KEGG pathways.

\subsection{Statistical Analysis}

Student's t-test was used to compare values between two independent groups.

\section{Results}

\subsection{Included Independent Studies}

A total of 68 studies were recorded using PubMed, EMBASE and web of science. 38 of which were excluded after screening the titles and abstracts, and 20 studies were excluded after reading the full text based on the inclusion and exclusion criteria, only 10 independent studies were included in this systematic analysis. The detailed workflow used in our analysis was shown in Figure 1. The detailed characteristics of each study are given in Table 2 in descending order of their year of publication.

\subsection{Differentially Expressed miRNAs}

These 10 studies from 10 different groups with different platforms, different number of samples, and various statistical analyses had been employed for microRNA profiling analysis to compare melanoma tissue with corresponding noncancerous skin tissue. The number of differentially expressed microRNAs ranges from 4 to 53 . A total of 303 differentially expressed miRNAs were reported in the 10 profiling studies. 171 microRNAs are downregulated in melanoma, and 132 microRNAs are up-regulated. Among the 171 down-regulated miRNAs, two of them were reported in 5 microarray studies (miRNA-204 and miRNA-125b), five miRNAs were reported in four profiling studies (miRNA-141, miRNA-149, miRNA-224, miRNA-200b, miRNA-200c). Among the 132 upregulated miRNAs, there was only one different miRNA in 5 studies (miRNA-21), three studies were reported 


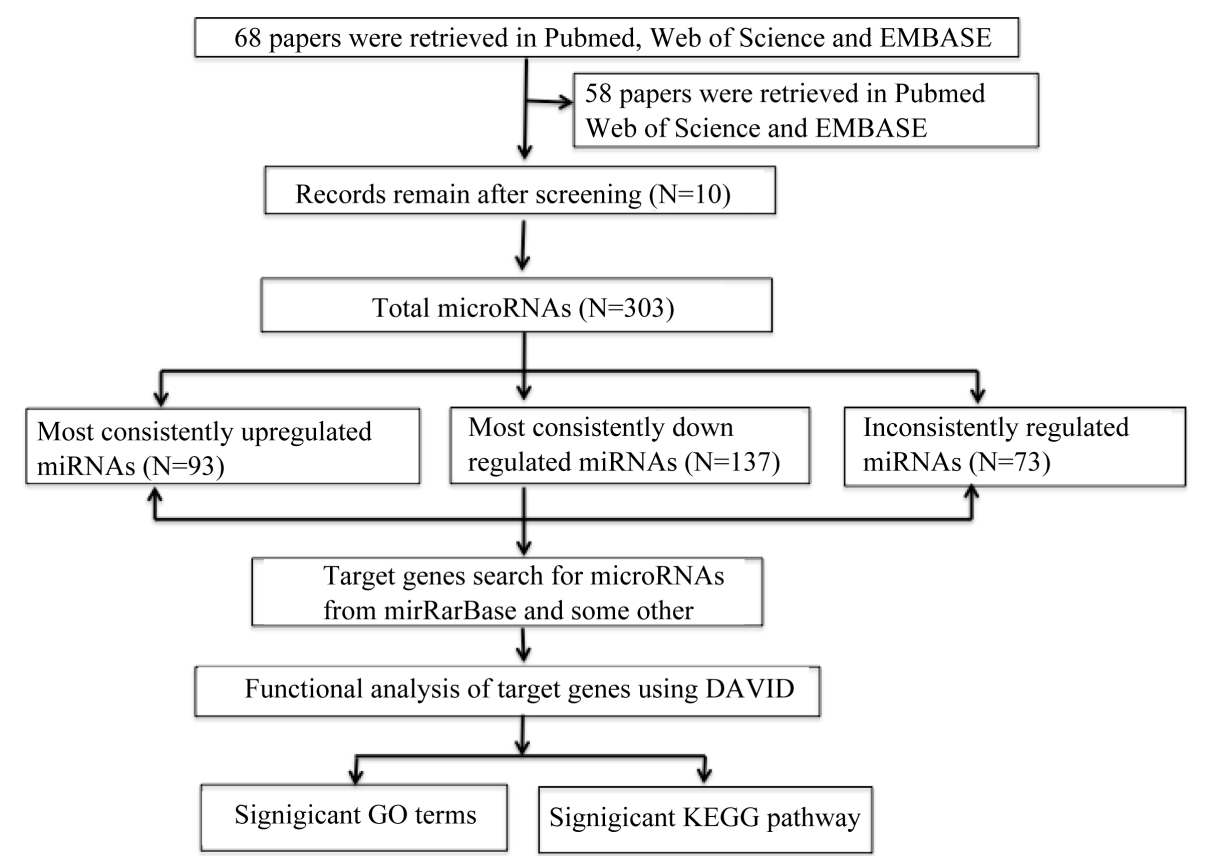

Figure 1. Flowchart for this systematic analysis.

Table 2. Characteristics of miRNAs datasets in hepatocellular carcinoma.

\begin{tabular}{|c|c|c|c|c|c|c|c|c|}
\hline Author & Region & Year & Platform & $\begin{array}{l}\text { Number of } \\
\text { Tissues }\end{array}$ & $\begin{array}{l}\text { Altered } \\
\text { miRNAs }\end{array}$ & Criteria & $\begin{array}{c}\text { Up-Regulated } \\
\text { miRNAs } \\
\text { in HCC }\end{array}$ & $\begin{array}{l}\text { Down-Regulated } \\
\text { miRNAs in HCC }\end{array}$ \\
\hline Qi & China & 2014 & GPL9052 Illumina & $31(7 / 24)$ & 4 & $\mathrm{p}<0.05$ & 4 & \\
\hline Sand & Germany & 2013 & Agilent Microarray & $42(21 / 21)$ & 49 & $\mathrm{p}<0.05$ & 21 & 28 \\
\hline $\mathrm{Xu}$ & UK & 2012 & Illumina microarray & $32(21 / 21)$ & 24 & $\mathrm{p}<0.05$ & 10 & 14 \\
\hline Poliseno & USA & 2012 & $\begin{array}{l}\text { miRCURY LNA } 11.0 \\
\text { array (Exiqon) }\end{array}$ & $91(82 / 9)$ & 27 & $\mathrm{p}<0.05$ & & 27 \\
\hline Yang & China & 2011 & LuxScan 3.0 & $8(4 / 4)$ & 7 & ? & 5 & 2 \\
\hline Caramta & USA & 2010 & Agilent Microarray & $19(16 / 3)$ & 54 & $\mathrm{p}<0.01$ & 48 & 6 \\
\hline Segura & USA & 2010 & Agilent Microarray & $118(59 / 59)$ & 12 & $\mathrm{p}<0.05$ & & 12 \\
\hline Chen & Canada & 2010 & Agilent Microarray & $16(8 / 8)$ & 31 & $\mathrm{p}<0.05$ & 13 & 18 \\
\hline Philippidou & Luxemburg & 2010 & uParaflo microarray & $40(20 / 20)$ & 45 & $\mathrm{p}<0.05$ & 25 & 20 \\
\hline Schultz & Germany & 2008 & ABI 7700 systerm & $20 \mid(10 / 10)$ & 72 & $\mathrm{p}<0.05$ & 6 & 66 \\
\hline
\end{tabular}

in 4 profiling studies (miRNA-146b, miRNA-17 and miRNA-18a). Here, consistently expressed miRNAs, the corresponding microarray study and the total number of tissue samples were shown in Table 3 . We also noticed that these selected miRNAs differentially expressed in a very inconsistent manner in 10 miRNA profiling studies. For example, in the groups of inconsistently reported miRNAs, miRNA-211 was reported down-regulated in three studies but up-regulated in two studies; miRNA-199a was increased in one study but down-regulated in 1 study (Table 4), respectively.

\subsection{Validation of the Selected miRNAs in Melanoma Patients}

To validate the consistency and direction of the eleven most consistently reported miRNAs (miRNA-204, miRNA125b, miRNA-141, miRNA-149, miRNA-224, miRNA-200b, miRNA-200c, miRNA-17, miRNA-21, miRNA146b and miRNA-18a), the expression of these miRNAs in melanoma biopsies and adjacent noncancerous tissues 
Table 3. Consistently reported up-/down-regulated miRNAs in profiling studies (Melanoma tissue vs. Normal).

\begin{tabular}{ccc}
\hline miRNAs & Frequence (references) & Number of tissue samples (melanoma/healthy) \\
\hline Up-regulated miRNAs & $5(16 ; 17 ; 19 ; 20 ; 24)$ & \\
miR-21 & $4(16 ; 20 ; 21 ; 22)$ & $100(52 / 48)$ \\
miR-17 & $4(16 ; 17 ; 21 ; 22)$ & $188(81 / 107)$ \\
miR-146b & $4(16 ; 17 ; 19 ; 22)$ & $136(68 / 68)$ \\
miR-18a & & $136(68 / 68)$ \\
Down-regulated miRNAs & $5(15 ; 16 ; 18 ; 20 ; 22)$ & \\
miR-204 & $5(15 ; 16 ; 18 ; 19 ; 22)$ & $90(47 / 43)$ \\
miR-125b & $4(15 ; 16 ; 21 ; 22)$ & $118(59 / 59)$ \\
miR-141 & $4(15 ; 16 ; 21 ; 22)$ & $108(54 / 54)$ \\
miR-149 & $4(15 ; 19 ; 21 ; 22)$ & $108(54 / 54)$ \\
miR-224 & $4(15 ; 16 ; 21 ; 22)$ & $132(54 / 78)$ \\
miR-200c & $4(15 ; 16 ; 21 ; 22)$ & $108(54 / 54)$ \\
miR-200b & $4(15 ; 16 ; 22)$ & $108(54 / 54)$ \\
miR-23b & $4(21 ; 22 ; 23)$ & $78(39 / 39)$ \\
miR-455 & & $190(95 / 95)$ \\
\hline
\end{tabular}

Table 4. Inconsistency of differentially expressed miRNAs.

\begin{tabular}{|c|c|c|}
\hline miRNAs & N\# of in up-regulated miRNAs & $\mathrm{N \#}$ of in down-regulated miRNAs \\
\hline miR-9 & 1 & 1 \\
\hline miR-17 & 1 & 5 \\
\hline miR-21 & 1 & 5 \\
\hline miR-28 & 2 & 1 \\
\hline miR-96 & 1 & 1 \\
\hline miR-107 & 1 & 1 \\
\hline miR-132 & 1 & 1 \\
\hline miR-140 & 1 & 1 \\
\hline miR-143 & 1 & 1 \\
\hline miR-146 & 1 & 1 \\
\hline miR-155 & 1 & 1 \\
\hline miR-185 & 1 & 2 \\
\hline miR-186 & 1 & 2 \\
\hline miR-194 & 1 & 1 \\
\hline miR-211 & 3 & 2 \\
\hline $\operatorname{miR}-219$ & 1 & 1 \\
\hline miR-323 & 1 & 2 \\
\hline miR-342 & 1 & 1 \\
\hline miR-494 & 1 & 1 \\
\hline miR-768 & 1 & 1 \\
\hline miR-106a & 1 & 1 \\
\hline miR-199a & 1 & 1 \\
\hline miR-26a & 1 & 1 \\
\hline miR-27a & 1 & 1 \\
\hline miR-29c & 1 & 1 \\
\hline
\end{tabular}


were compared in 6melanoma patients using real-time PCR with specific primers (Table 1). The results showed that the expressions of miRNA-21, miRNA-146b and miRNA-18a were up-regulated in melanoma tissues (no significant different was seen for miRNA-17) (Figure 2), whereas in selected nine down-regulated miRNAs, five of them (miRNA-204, miRNA-125b, miRNA-141, miRNA-200b, miRNA-200c) were down-regulated significantly in melanoma patients (Figure 3), but two of them (miRNA-149, miRNA-224) did not see significant different expression, which were inconsistent with those of the original corresponding profiling studies.

\subsection{Primary miRNAs Analysis}

The identified and selected miRNAs all play specific roles in skin homeostasis, or pathogenesis of melanoma. Particularly, all these miRNAs are either oncogenic miRNAs or tumor suppressors. Specifically, miRNA-21, miRNA-146b and miRNA-18a are oncogenic miRNAs (oncomir), and miRNA-204, miRNA-125b, miRNA-141, miRNA-200b and miRNA-200care tumor suppressors. We need to point out that these selected oncomires and tumor suppressors are differentially expressed only in moderate number of profiling studies (less than 5 or 4 times), implying that miRNA plays marginal roles in the tumorigeneisis and development of melanoma.

\subsection{Target Genes of Differentially Expressed microRNAs}

After we identified the most consistently differentially expressed microRNAs that were either down-regulated
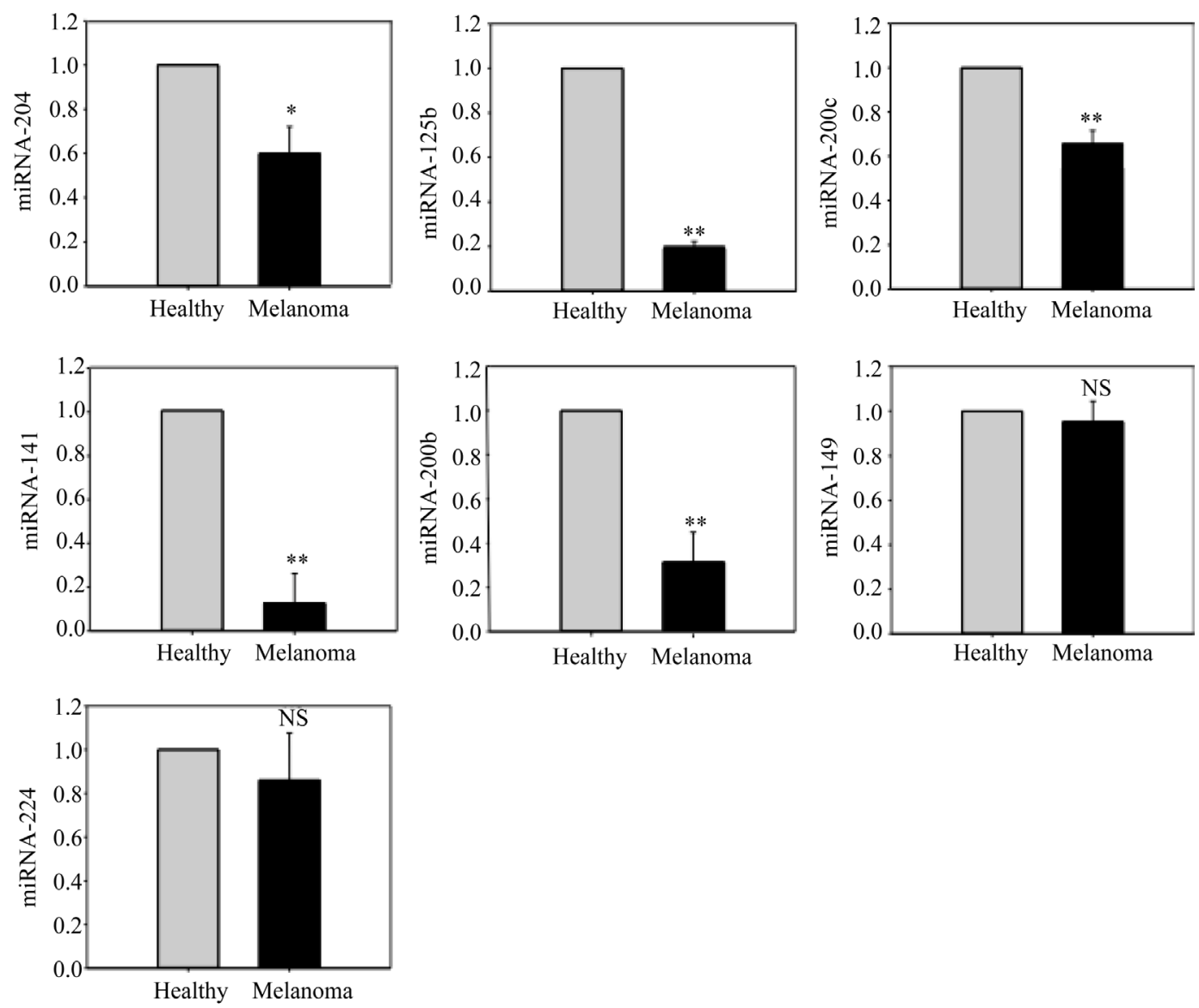

Figure 2. Real-time PCR analysis of some most consistent down-regulated expressed miRNAs. Expression levels of miR-204, miR-125b, miR-149, miR-141, miR-200b, miR-200c, and miR-224 in melanoma and adjacent noncancerous tissue samples were analyzed by real time PCR, U6 here functions as an internal control. The expressions of miR-204, miR-125b, miR-141, miR-200band miR-200cwere significantly decreased in melanoma tissues, while the expressions of miR-149 and miR-224 were significantly reduced. Here, values represent means \pm S.E. of three determinations. *, $\mathrm{p}<0.05 ; * *, \mathrm{p}<0.01$. 

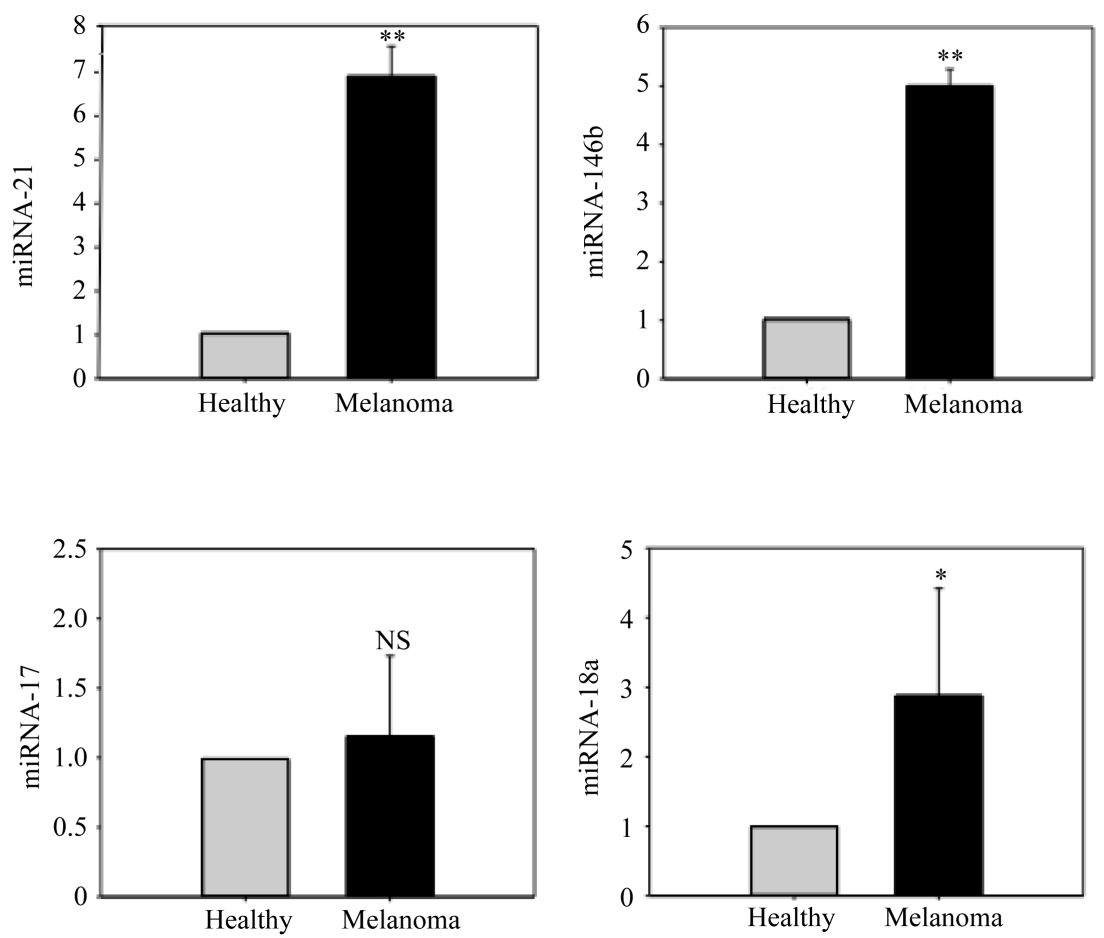

\begin{abstract}
Figure 3. Real-time PCR analysis of some most consistent up-regulated expressed miRNAs. Expression levels of miR-17, miR-146b, miR-21 and miR-18a in melanoma and adjacent noncancerous tissue samples were analyzed by real time PCR, U6 here functions as an internal control. The expressions of miR-21, miR-146b and miR-18a were significantly higher in melanoma tissues, while the expression of miR-17 was significantly reduced. Here, values represent means \pm S.E. of three determinations. *, $\mathrm{p}<0.05 ; * *, \mathrm{p}<0.01$.
\end{abstract}

or up-regulated in melanoma, we screened the their potential target genes with programs such as miRTarBase (mirtarbase.mbc.nctu.edu.tw), microRNA.org and TargetScanHuman 6.2, focusing on the genes which were confirmed either by real time PCR, western blot, microarray or luciferase assay. As a result, we identified 211 and 318 target genes (519 in total, some target genes are shared by two different kinds of miRNAs, reasons are to be defined) corresponding to those down-regulated and up-regulated miRNAs in melanoma (Table 5 and Table 6), respectively.

\title{
3.6. Enrichment Analysis Result
}

We used DAVID program to build up the molecular networks of down-regulated and up-regulated microRNAs target genes. The top GO terms (18 for up-regulated miRNAs and 17 for down-regulated miRNAs) and KEGG pathways showing significant association with target genes were listed with GO terms, KEGG pathway, number of genes in the terms and number of genes in the pathways (Figures 4-9). The important GO terms were critically involved in the regulation of regulation of transcription, metabolic process for down-regulated miRNAs (Figure 4), and programmed cell death for up-regulated miRNAs (Figure 6), as shown all these functions in combined total miRNAs (Figure 8). KEGG pathways (KEGG pathways in melanoma from target genes of down-regulated miRNAs (Figure 5) and up-regulated miRNAs (Fgiure 7) are very similar and largely overlap with relevant signaling pathways and cellular functions, even though the target genes themselves may function quite differently. The KEGG pathways in melanoma from entire target genes (Figure 8), similar with the ones from up-regulated or down regulated genes, were combined with various types of pathways related to tumorigenesis of cancers, like apoptosis (such as Fas, TNF and IRAK) (Supplemental data S1), p53 signaling pathway (such as p21, cyclin E and p53) (Supplemental data S2), cell cycle (such as Smad4, ARF and c-Myc) (Supplemental data S3), regulation of actin cytoskeleton (Raf, Ras and Rho) (Supplemental data S4), MAPK (such as Raf, Ras and EGFR) (Supplemental data S5), toll-like receptor signaling pathway (TLR2, TLR4 and IRAK) (Supplemental data S6), mTOR signaling pathway (such as IGF, AKT and HIF1a) (Supplemental data S7) and 
Table 5. Selected up-regulated miRNAs and their predicated target genes.

\begin{tabular}{|c|c|}
\hline $\begin{array}{l}\text { Up-Regulated } \\
\text { miRNAs }\end{array}$ & Predicted Target \\
\hline miR-21 & $\begin{array}{l}\text { BTG2, CDKAP1, PDCD4, LRRFIP1, CDC25A, PELI1, PDCD4, TP63, PTEN, HNRPK, TGFBR2, TGFBR2, } \\
\text { SMARCA4, SPRY1/2, PRARA, TIMP3, RHOB, MSH2/6, ANP32A, BCL2, ANP32A, BMPR2, MARCK5, } \\
\text { ANKRD46, RECK }\end{array}$ \\
\hline miR-17 & $\begin{array}{l}\text { ZNFX1, CCL1, GPR137B, NABP1, NPAT, YES1, JAK1, PTEN, CDKN1A, PTPRO, PKD2, BCL2L11, E2F1, } \\
\text { MAP3K12, BCL2, MEF2D, RUNX1, APP, ICAM1, VEGFA, MAPK9, DNAJC27, FBXO31, TGFBR2, TNFSF12, } \\
\text { MUC17, BMPR2, VIM, CCND1, MYC, NCOA3, THBS1, SMAD4, ICAM1, SELE, SOD2, GPX2, TXNRD2, } \\
\text { CCND2, E2F3, RB1, RBL1, RBL2, WEE1, TGFBR2, RND3, SMURF1, TCF3, HSPB2, MMP2, HBP1, GALNT7, } \\
\text { SIRPA, DYNC1LI2, PLEKHA3, BRMS1L, ZNFX1, MYT1L, EPHA4, ENPP5, SLITRK3, RPS6KA5, SERF1B, } \\
\text { CAMTA1, C14ORF28, ANUBL1, RUFY2, SUV420H1, GNPDA2, SCN1A, SERF1A, RPGR, LHX8, PKD2, GPR6, } \\
\text { RALYL, GPR137C, ATG7, SACS, VSX1, PCDHA13, PCDHA5, PCDHA4, ANKRD44, MYEF2,TRIP11, } \\
\text { ARHGAP12, TANC1, STAT3, NBEA, NR4A2, ZNF367, CAMK2N1, ZNF800, MYNN, PDCD1LG2, PRDM6, } \\
\text { ARID4A, PFN2, FTSJD1, AGGF1, PCDHA12 }\end{array}$ \\
\hline miR-146b & $\begin{array}{l}\text { CXCR4, CFH, IRAK2, TLR2, FADD, TRAF6, IRAK1, IRCK1, ROCK1, BRCA2, BPCA1, BRCA1, FAF1CCNA2, } \\
\text { PA2G4, IL8, NFKB1, CDKN1A, EGFR, MTA2, MMP16, KIT, Card10, Scube2, TRAF6, IRAK1, CD40LG, PDGFB, } \\
\text { FAS, CDKN3, KIF22, ERBB4, SMAD4, TLR4, WASF2, BGLAP, SPP1, SLPI, L1CAM, NOVA1, RHOXF2B, } \\
\text { RHOXF2, TPM1, TRAF6, TRAF6, IGSF1, PCGF5, PCGF5, ZNF90, TMEM185B, ITCH, LIN52, PHC1, FAM153B, } \\
\text { ABCD3, CMAH, LRRTM3, PMS1, TFAP2D, LRCH1, MGC11082, TMEM19, ADRB2, RASGRP1, FBXL3, } \\
\text { LCA5L, UBE2J1, RAB7L1, RAK1, UHRF1, SLC16A14, TIMELESS, AGMAT, CCDC150, CADM2, RFTN2, } \\
\text { SLC1A1, TRDN, FLJ10489, TXNDC5, C10orf4, RNF148, RHOBTB3, DPY19L2P1, DPY19L2, BLZF1, PPM1K, } \\
\text { DPY19L2P4, ARF6, RUNDC2B, RUNDC2C, PAK7, ANKRD11, LOC203274, SLC6A14, ARNT, CAMK2G, } \\
\text { GBA2, TCHP, CCNB3, MORF4L2, PTPN12, TSC22D2, SULT2A1, BTBD11, PRKCH, TTC38, CCNE1, } \\
\text { FAM160B1, KALRN, ORC4L, C14orf129, STRN, DYNLL1, PIWIL4, TET2, CMTM1, ITM2B, C6orf94, SIGLEC5, } \\
\text { HLA-F, CPA6, COPZ1, ACAD8, PARK7, MBNL1, UBE2D2, ABCD3, DECR1, RBM15, OXR1, DNM1L, IL1A, } \\
\text { TUBD1, CIRH1A, FAM35B2, HIF3A, CPEB4, TTLL11 }\end{array}$ \\
\hline miR-18a & $\begin{array}{l}\text { KRAS, ESR1, PTEN, Runx1, CTGF, NCOA3, CCNL1, CSRNP3, THRA, TNFSF11, Myc, Prmt5, NR3C1, TSC22D3, } \\
\text { Prmt5, SMAD4, HSF2, ATM, Smad2, Smad4, NEDD9, CDK19, DICER1, SMAD3, Pten, ERLIN1, OTX2, ENTHD1, } \\
\text { GLRB, BEND6, BBX, INADL, CAB39, NEDD9, HIF1A, TXK, DSC1, DIP2C, BTG3, MAP3K1, SCD5, PRPF4B, } \\
\text { TMEM2, RIMS2, AKAP7, PRDM6, RAD51L1, HCFC2, ITM2B, CASC2, PFN2, RMST, CDS2, MTCP1, TMEM64, } \\
\text { BEX1, NAE1, NCOA1, ZNF367,PHF20L1, SAR1A, C22orf31, C5orf30, FAM3C, MAP7D1, RFC4, PPIP5K2, } \\
\text { RPL23AP82, KLHL20, PTPMT1, TRPC4, VPS13A, SH3BP4, RNF145, PASK }\end{array}$ \\
\hline
\end{tabular}

WnT signaling pathway (Wnt, Frizzled and RhoA) (Supplemental data S8). In the context of tumorigenesis, all these pathways work synergistically to evade apoptosis by regulation of transcription of these target genes, which in turn regulates relevant signaling pathways.

\section{Discussion}

In the current study, we established the concept, by conducting a systematic analysis, which only a few of consistently differentially expressed miRNAs may be of significance for tumorigenesis and development of melanoma. The potential targets of these selected miRNAs conduct the signaling pathways critical for programmed cell death and the regulation of transcription. Together, this research shed some light on the potential of certain miRNAs as biomarkers and therapeutic target for melanoma.

Many conventional tumor biomarkers, such as ovarian cancer biomarker CA-125 and melanoma biomarker S-100, have encountered some limitation like poor sensitivity of specificity, for example, only $50 \%$ of patients with early stage ovarian cancer have elevated levels of CA125; specificity of S-100 for melanoma is about 75\% [30]. Thus, there is a critical need for reliable biomarkers that allow diagnosis with high sensitivity and precise monitoring the pathogenic process and therapeutic alternatives of melanoma. Many miRNAs are deregulated significantly even in early stages of tumor development and involved in tumorigenesis and metastasis of many caner categories, exhibiting their diagnostic and therapeutic potential utility. However, as many miRNA profiling studies on melanoma were performed on different platforms and very different processing methods by different investigators, there lacks the common concert among these studies, consequently, which ended within consistent miRNAs expression profile [15]-[25].

Based on our systematic analysis, these oncomirs and tumor suppressors were consistently and differentially expressed in relative less investigations (no more than 5 profiling studies), suggesting that miRNAs may only 
Table 6. Selected down-regulated miRNAs and their predicated target genes.

\begin{tabular}{ll}
\hline $\begin{array}{l}\text { Down-Regulated } \\
\text { miRNAs }\end{array}$ & Predicted Target \\
\hline & MEIS1, HOXA10, BCL2, TGFBR1, TGFBR2, SNAI2, Runx2, MEIS2, SNAI1, Mmp9, Aurkb, Hoxb7, SPDEF, \\
& THRB, CDX2, CDX2, BCL2L2, BIRC2, EDEM1, EZR, FARP1, FZD1, IL11, M6PR, RAB22A, RAB40B, SERINC3, \\
& SERP1, TCF12, TCF4, FOXC1, MAP1LC3B, CREB5, ELOVL6, RUNX2, SOX4, EFNB2, ALPL,SOST,SLC37A3, \\
miR-204 & BPY2, ACSL4, ZNF638, GRIA2, ZCCHC10, TARDBP, AP1S2, RHOBTB1, HELLS, GRIA4, LRIT3, PRAMEF8, \\
& JRKL, ACSM2A, CHEK1, PHOX2B, ACSM2B, C2orf68, C9orf72, ENTPD5, CNOT10, MLLT3, MAPRE2, \\
& PRAMEF7, ELOVL6, BRWD1, PRO0628, ATP10B, LOC284294, EGR1, NR4A2, FSIP2, NDRG3, HDAC9, \\
& C2orf56, SS18, NUDT13, LOC253724, TRMT11, TMEM57, BHLHE22, ZFP91-CNTF, ZFP91-CNTF, SIX1, \\
& GATAD1, GPR6, RAP2C, B3GNT5, KIAA0776, PTGR1
\end{tabular}

BMPR1B, EIF4EBP1, HMGA2, HMGA1, GLI1, NKIRAS2, TP53, SMO, VDR, SGPL1, BAK1, ERBB3, ERBB2, BMF, KLF13, NTRK3, LIN28A, CBFB, AKT1, CYP24A1, RAF1, SMO, PRDM1, IRF4, GRIN2A, CDKN2A, LIN28A, MAP2K7, JUB, KRT7, TNF, TP53INP1, E2F3, TRIM71, IGF2, LIN28B, BAK1, BBC3, BMF, KLF13, TEF, STAT3, BAK1, JUN, JUND, PPP1CA, PPKRA, PRKRA, PPP1CAB, SRF, NKX2-5, PRPF8, BCL2, ETS1, RPS6KA1, TNFAIP3, PIGF, BCL3, TBC1D1, DGAT1, FGFR2, SUV39H1, ARID3B, SMAD4, MCL1, IL6R, STARD13, ABTB1, CBFB, HK2, MMP13, SNAI1, MAPK14, MUC1, NES

ZEB2, ZEB1, DLX5, BAP1, Dlx5, Cdh11, Zeb2, Zeb1, Stk3, Klf5, KLF5, STK3, TGFB2,SFPQ, CLOCK, BRD3, UBAP1, PTEN, ZFPM2, TRAPPC2P1, EIF4E, CTBP2, CDYL, ACVR2B,MAPK14, PPARA, NR0B2, YWHAG, Elav14, MAPK9, TFDP2, E2F3, SHC1, VAC14, TCF7L1, ELMO2, RASSF2, KLHL20, RIN2, SEPT7, HOXB5,

$\operatorname{miR}-141$ ERBB2IP, KLF11, PTPRD, WDR37, AP3S1, RANBP6, ARPC5, WTAP, TAC1, NRP1, ALS2, PITX2, MTF2, SELE, ITGA6, ZFR, IRF2BP2, IRF2BP2, PCDH9, LMO3, KCNIP4, SLC18A2, TRAM1, LHFP, IPO5, YY1, UFM1, C11orf54, LEPR, FAM188A, MYH10, LGR4, ZNF248, HS6ST2, ELAVL2, BBS10, SNRPB2, KIDINS220, ANKS1B, NBPF16, NBPF8, PRKD1, ATL1, GLCCI1, CHD9, TSHZ3, MARCH7, LBR, UNC5C, SP4, MYBL1, NBPF11, MAP2K4

Aicda, AKT1, E2F1, SP1, FOXM1,FRMD7, PPIP5K1, RSBN1L, ERBB3, KIAA1467, C17orf81, BEYLA, CAV2, DYNLRB1, POLR2L,HINFP, EGLN3, MOGAT2, BIRC5, MRPS22, CACHD1, PPAP2B, CCDC109A, RNF2,

miR-149 YJEFN3, NISCH, RUNDC2C, BRPF3, SPEF2, NUCB2, KCNIP1, HSFYL1, CHEK1, SH3GL3, STRADB, TOP1, HSD17B4, NAP1L1, MAGEB18, ZMYM6, STRA6, RAP1BL, RAP1B, ZNF385D, PRDM2, IL6, RAP1A, KCNMA1, HNRNPA1, PHLPP2, UPF2, SSBP1, CCNI, ODZ3, CCDC45

TUBB3, BMI1, SIP1, BAP1, ZEB2, ZEB1, Zeb1, Zeb2, FN1, ZFPM2, UBE2I, JAG1, PTPN13, Flt1, RNF2, RCOR3, BRD7, ACVR2B, Mapk14, MSN, NTRK2, ERRFI1, FHOD1, PPM1F, CCNE2, XIAP, BCL2, TIMP2, FBLN5, VEGFA, NCAM1, Vldlr, Reln, IKBKB, FLT1, KLF9, TBK1, PMAIP1, NTF3, LPAR1, EDNRA, RHOA, KLHL20,

miR-200c PTPRD, ELMO2, ERBB2IP, WDR37, VAC14, TCF7L1, RASSF2, HOXB5, RIN2, KLF11, SEPT7, SHC1, CGRRF1, QKI, WIPF1, LCA5, LOX, ERRFI1, RBM46, LEPR, DNA2, PTPN22, CCNJ, FOXF1, TSGA14, NRG1, SEC23A, RECK, VASH2, KIAA1244, NOG, MARCKS, ASF1A, A2BP1, LHFP, RAP1BL, RAP1B, ZNF423, C9orf93, ZNF532, LRP1B, MEX3B, ELAVL2, APOO, ZDHHC17, INTS8, MSN, NOVA1, CDK17, RAP2C, ZNF292, GPR158, LBR, GPM6A, TFAP2A, KCTD8, WNT16, SULF1, HSPA9, WIF1

PTPN12, ZEB2, BAP1, ZEB1, RERE, Zeb2, Zeb1, ets1, gata4, fn1, wasf3, zfpm2, matr3, rnf2, bml1, ezf3, vegfa, FLT1, KDR, RND3, CCNE2, BCL2, XIAP, SMAD2, CREB1, KLHL20, ELMO2, PTPRD, ERBB21P, WDR37, TCF7L1, VAC14, HOXB5, RIN2, RASSF2, KLF11, SEPT7, SHC1

\begin{tabular}{|c|c|c|c|}
\hline Torm & जै RT & Gones & Count $=$ \\
\hline regulation of transcription from RNA polymerase II promoter & $\underline{\mathbf{R T}}$ & $\overline{\mathbf{a}}$ & 51 \\
\hline regulation of transcription, DNA-dependent & $\underline{\mathbf{R T}}$ & $\overline{\mathbf{a}}$ & 85 \\
\hline positive regulation of macromolecule metabolic process & $\underline{\mathbf{R T}}$ & $\overline{\mathbf{a}}$ & 55 \\
\hline regulation of RNA metabolic process & $\underline{\mathbf{R T}}$ & $\overline{\mathbf{a}}$ & 85 \\
\hline regulation of transcription & $\underline{\mathbf{R T}}$ & $\overline{\mathbf{a}}$ & 106 \\
\hline regulation of cell proliferation & $\underline{\mathbf{R T}}$ & $\overline{\mathbf{a}}$ & 50 \\
\hline transcription & $\underline{\mathbf{R T}}$ & $\overline{\mathbf{E}}$ & 80 \\
\hline cell surface receptor linked signal transduction & $\underline{\mathbf{R T}}$ & $\bar{\Sigma}$ & 58 \\
\hline
\end{tabular}

Figure 4. GO analysis of target genes from most up-regulated miRNAs in selected profiling studies. Predicted target genes are mainly involved in cancer related cellular process including programmed cell death and metabolic process; further, these involvement are probably through the regulation of transcription regulation. 


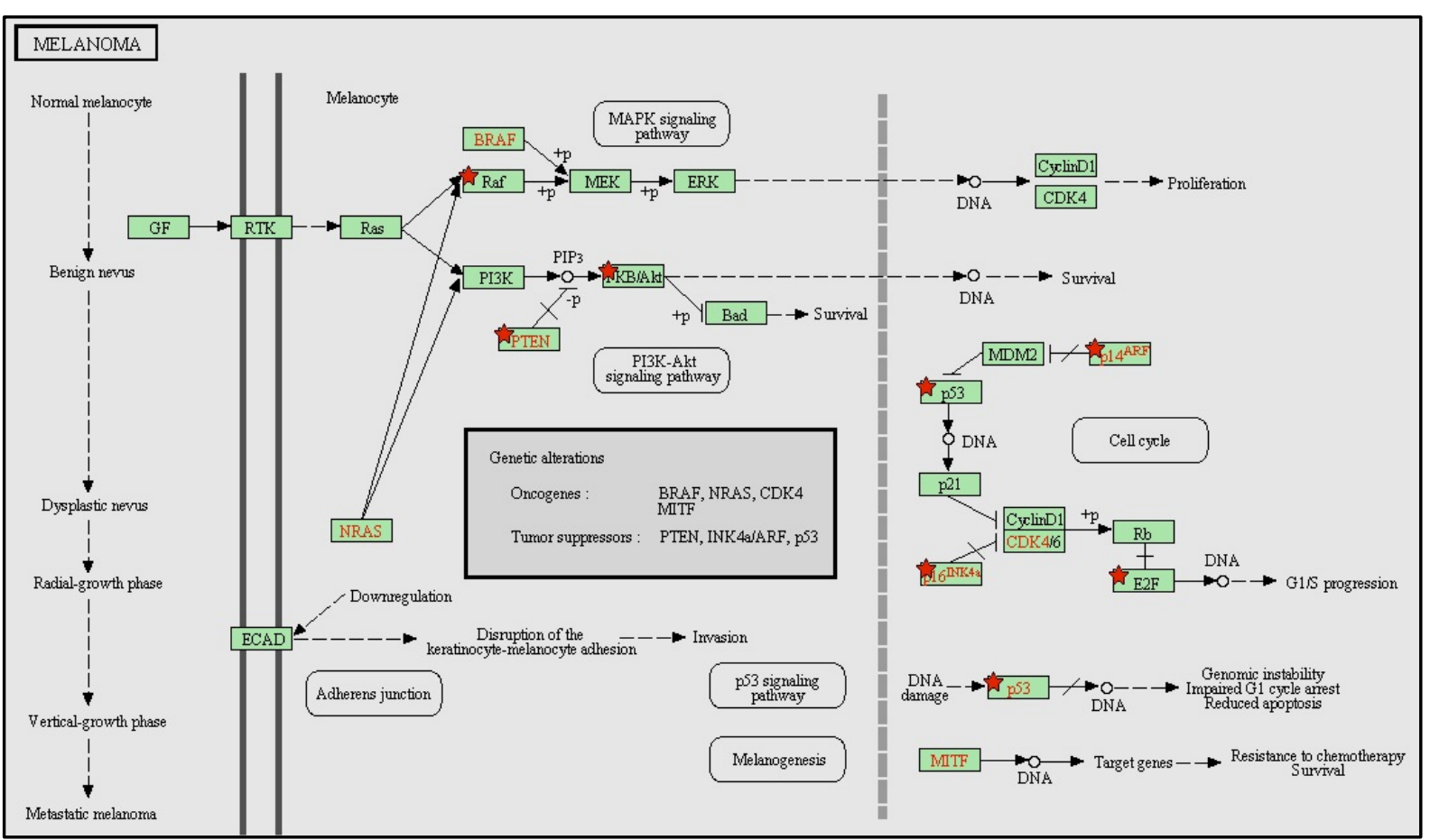

Figure 5. KEGG pathway (pathways in cancer) analysis with predicated target genes from up-regulated miRNAs. These pathways in cancer are a combination of many different but relevant pathways, such as MAPK signaling pathway and cell cycle signaling pathway, etc. Green boxes with red star represent the potential genes targeted by selected miRNAs.

\begin{tabular}{|ll|l|l|}
\hline \multicolumn{1}{c}{ Torm } & A RT & Gonos & Count \\
\hline regulation of programmed cell death & RT & 36 \\
\hline regulation of cell death & $\underline{\text { RT }}$ & 36 \\
\hline regulation of apoptosis & $\underline{\mathbf{R T}}$ & 35 \\
\hline intracellular signaling cascade & $\underline{\mathbf{R T}}$ & 41 \\
\hline positive regulation of macromolecule metabolic process & $\underline{\mathbf{R T}}$ & 31 \\
\hline regulation of transcription & $\underline{\mathbf{R T}}$ & 57 \\
\hline regulation of RNA metabolic process & $\underline{\mathbf{R T}}$ & 41 \\
\hline regulation of transcription, DNA-dependent & RT & 40 \\
\hline
\end{tabular}

Figure 6. GO analysis of target genes from most down-regulated miRNAs in selected profiling studies. Predicted target genes are mainly involved in cancer related cellular process including programmed cell death, metabolic process; further, these involvements are probably through the regulation of transcription regulation.

play marginal roles in the tumorigenesis and development of melanoma, which is concert with the fact that environmental factors like UV plays important roles in the onset of melanoma. Here, we found that three of the selected miRNAs (miRNA-21, miRNA-17, miRNA-146b and miRNA-18a) are enhanced, displaying oncogenic characteristics (oncomir), and seven of screened miRNAs (miRNA-204, miRNA-125b, miRNA-141, miRNA200b and miRNA-200c) decreased markedly, functioning as tumor suppressors. As an oncomir, miRNA-21 negatively regulates the expression of many different tumor suppressor genes, such as PTEN [31], Bcl-2 [32] and Il-12p35 [33] etc, by which is involved in the tumorigenesis of different cancers like colon [34], liver [31] and lung [35]. The involvement of miR-17 in pathogenesis of cancer is controversial and open to debate; miR-17 is up-regulated as oncomir in breast cancer in some groups [36]-[38], but act as tumor suppressor in breast cancer in another group [39], specifically, miR-17 inhibits tumor growth by stimulating T-cell mediated host immune response in melanoma [40]. In our systematic study, miR-125bperforms tumor-suppressor functions in several 


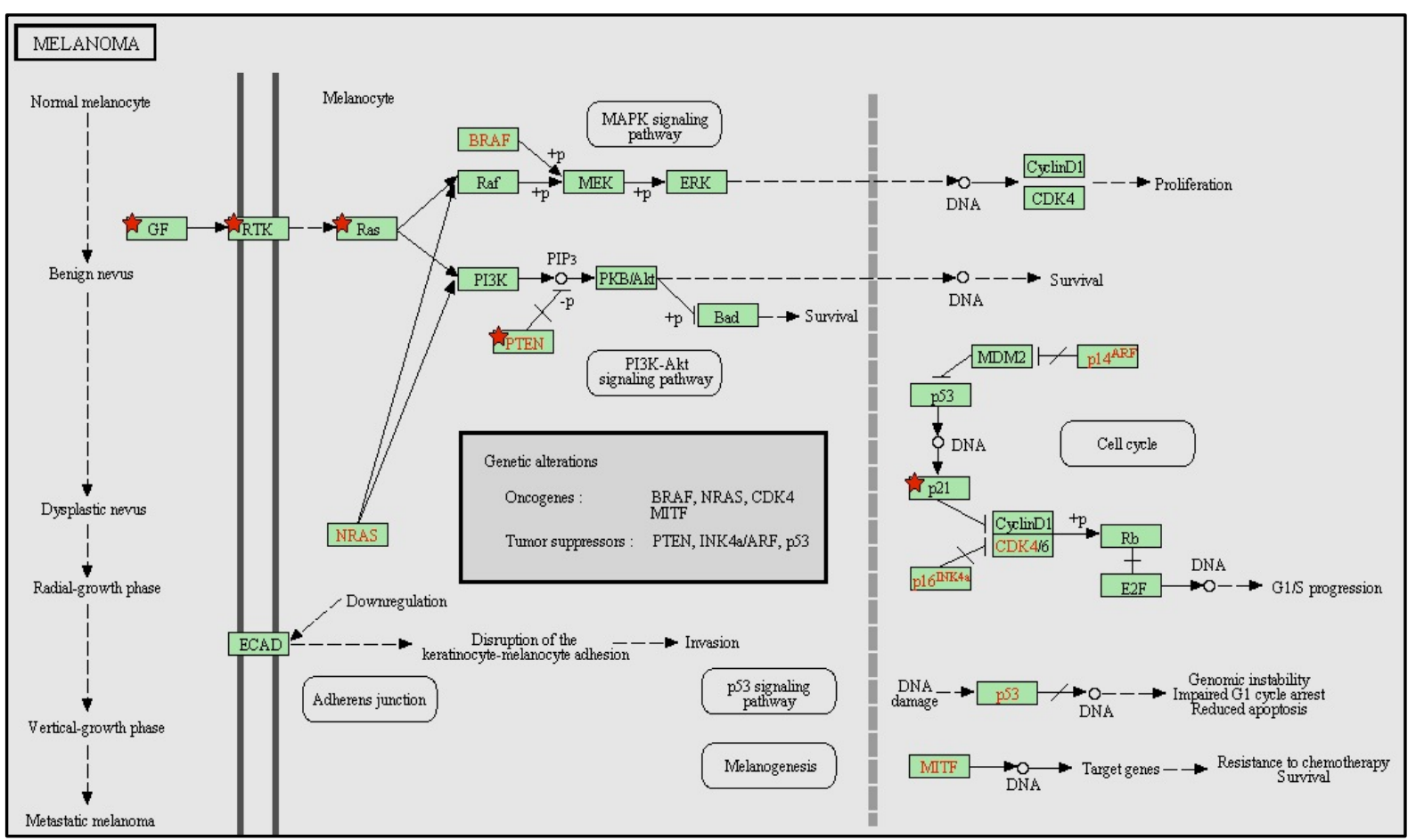

Figure 7. KEGG pathway (pathways in cancer) analysis with predicated target genes from down-regulated miRNAs. These pathways in cancer are a combination of many different but relevant pathways, such as MAPK signaling pathway, cell cycle signaling pathway, PI3K-Akt signaling pathway etc. Green boxes with red star represent the potential genes targeted by selected miRNAs.

\begin{tabular}{|c|c|c|c|}
\hline Torm & ज्ञ RT & Gones & Count - ve \\
\hline positive regulation of macromolecule metabolic process & $\underline{\mathbf{R T}}$ & $\bar{\equiv}$ & 83 \\
\hline regulation of programmed cell death & $\underline{\mathbf{R T}}$ & $\bar{\equiv}$ & 80 \\
\hline regulation of cell death & $\underline{\mathbf{R T}}$ & $=$ & 80 \\
\hline regulation of apoptosis & $\underline{\mathbf{R T}}$ & 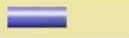 & 79 \\
\hline regulation of transcription & $\underline{\mathbf{R T}}$ & $\bar{\square}$ & 161 \\
\hline regulation of transcription, DNA-dependent & $\underline{\mathbf{R T}}$ & $\bar{\square}$ & 123 \\
\hline regulation of RNA metabolic process & $\underline{\mathbf{R T}}$ & $\overline{\square=}$ & 124 \\
\hline intracellular signaling cascade & $\underline{\mathbf{R T}}$ & $\bar{\square}$ & 83 \\
\hline transcription & $\underline{\mathbf{R T}}$ & $\overline{0}$ & 114 \\
\hline cell surface receptor linked signal transduction & $\underline{\mathbf{R T}}$ & $\bar{\square}$ & 80 \\
\hline
\end{tabular}

Figure 8. GO analysis of target genes from all the differentially expressed miRNAs in selected profiling studies. Predicted target genes are mainly involved in cancer related cellular process including programmed cell death, which is performed probably though cell transcription regulation.

cancers including melanoma, by suppressing melanoma cell proliferation and metastasis. miR-125b is downregulated in metastatic melanoma and controls melanoma progression by direct regulation of c-Jun protein expression [18] [41]. As a tumor suppressor, miR-204 was decreased in melanoma [42] and can inhibit cell proliferation by interfering with target gene IGFBP5 or RAB22A [43] [44].

miRNAs function as oncogenes or tumor suppressors by involving multiple molecular mechanisms. By enrichment analysis, we found these oncomirs and tumor suppressors can targets variety of substrate genes, it is of great significance to point out that these targets concentrate on the cancer-related cellular process, mainly including apoptosis, which is in concert with the fact that apoptosis plays checkpoint roles in tumorigenesis of any cancers [45] [46], which further highlight the importance of these miRNAs as potential biomarkers or therapeutic alternatives. KEGG pathways analysis by DAVID demonstrated that the target genes of these miRNAs 


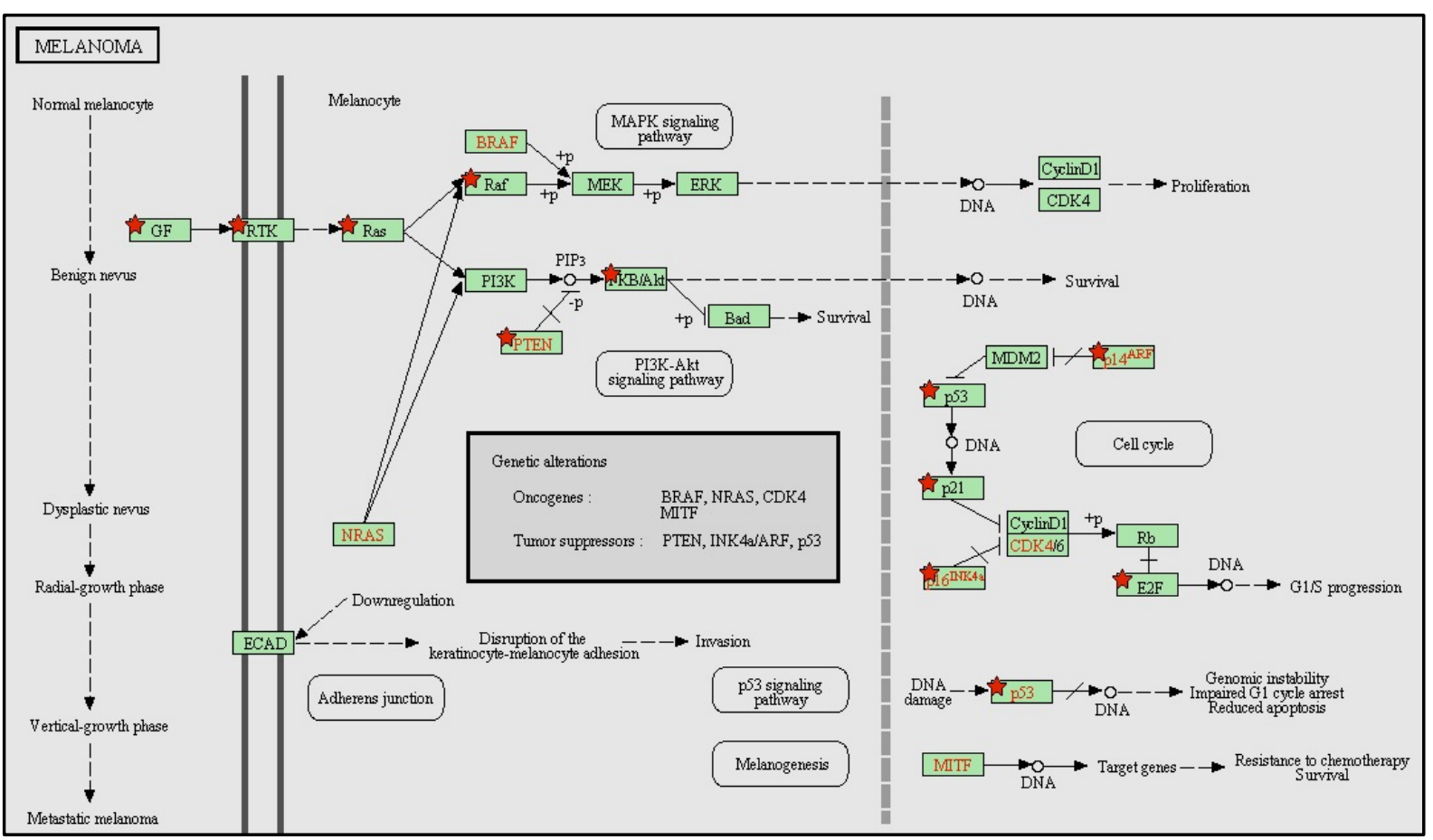

Figure 9. KEGG pathway (pathways in cancer) analysis with predicated target genes from all selected differentially expressed miRNAs. These pathways in cancer are a combination of many different but relevant pathways, such as MAPK signaling pathway, cell cycle signaling pathway and PI3K-Akt signaling pathway etc. Green boxes with red star represent the potential genes targeted by selected miRNAs.

built up a very rich networks, combined with different pathways and molecule-molecule interactions. Wnt signaling pathway is very important for the integrity of endothelial cells and for melanoma tissue invasion and metastasis, inhibition of mTOR signaling pathway can reduce cellular apoptosis; p53 signaling pathway contributes to inhibit apoptosis; MAPK signaling pathway are crucial for cell proliferation; All these pathways work synergistically to evade apoptosis and increase proliferation. Some other pathways related to block of differentiation, resistance to chemotherapy, failed repair of genes, insensitivity to anti-growth signals are also implied. All these miRNA/target genes related pathways mimic exactly the pathways involved in the tumorigenesis of melanoma, which make these miRNAs even more important as potential biomarkers and therapeutic candidates for clinical application. Extensive studies should focus these miRNAs to establish them as biomarkers for cancer detection and progression.

\section{Limitations}

Several limitations of this research should be considered when interpreting the results due to some unsolvable reasons. Firstly, our literature searching was depended on English databases only, as a result, language bias may present. Secondly, our study did not include all the populations (only Chinese, British, American, German, Canadian and Luxemburg), so the result may not be able to apply to other populations such as Latin American and African.

\section{Conclusion}

We performed comprehensive literature search in multiple databases by limiting publication language and date. By systematic analysis, we filtered the most consistently expressed 4 up-regulated and 7 down-regulated miRNAs, and further confirmed by real time PCR, specially, highlight would be given to miRNA-21, miRNA-204 and miR-125b since they consistently differentially expressed the most (5 studies respectively). By DAVID analysis, we showed that the target genes were mainly involved in the regulation of programmed cell death mediated by combined signaling pathways such as cell cycle signaling pathway. In short, these selected miRNAs 
are hopefully of significance by their potential as biomarkers and/or as therapeutic agents against melanoma.

\section{Acknowledgements}

This work was supported by grants from the Natural Science Foundation of China (81570479), the Climbing Program in Hangzhou Normal University-Phase II (PF14002004021) and the Program for Zhejiang Leading Team of Science and Technology Innovation (2011R50021).

\section{References}

[1] Cummins, D.L., Cummins, J.M., Pantle, H., Silverman, M.A., Leonard, A.L. and Chanmugam, A. (2006) Cutaneous Malignant Melanoma. Mayo Clinic Proceedings, 81, 500-507. http://dx.doi.org/10.4065/81.4.500

[2] Gray-Schopfer, V., Wellbrock, C. and Marais, R. (2007) Melanoma Biology and New Targeted Therapy. Nature, 445, 851-857. http://dx.doi.org/10.1038/nature05661

[3] Rubin, K.M. and Lawrence, D.P. (2009) Your Patient with Melanoma: Staging, Prognosis, and Treatment. Oncology (Williston Park), 23, 13-21.

[4] Bevona, C. and Sober, A.J. (2002) Melanoma Incidence Trends. Dermatologic Clinics, 20, 589-595. http://dx.doi.org/10.1016/S0733-8635(02)00037-2

[5] Segura, M.F., Greenwald, H.S., Hanniford, D., Osman, I. and Hernando, E. (2012) MicroRNA and Cutaneous Melanoma: From Discovery to Prognosis and Therapy. Carcinogenesis, 33, 1823-1832. http://dx.doi.org/10.1093/carcin/bgs205

[6] Siegel, R.L., Jemal, A. and Ward, E.M. (2009) Increase in Incidence of Colorectal Cancer among Young Men and Women in the United States. Cancer Epidemiology, Biomarkers \& Prevention, 18, 1695-1698. http://dx.doi.org/10.1158/1055-9965.EPI-09-0186

[7] Miller, A.J. and Mihm Jr., M.C. (2006) Melanoma. The New England Journal of Medicine, 355, 51-65. http://dx.doi.org/10.1056/NEJMra052166

[8] Bartel, D.P. (2004) MicroRNAs: Genomics, Biogenesis, Mechanism, and Function. Cell, 116, 281-297. http://dx.doi.org/10.1016/S0092-8674(04)00045-5

[9] Brennecke, J., Hipfner, D.R., Stark, A., Russell, R.B. and Cohen, S.M. (2003) Bantam Encodes a Developmentally Regulated microRNA that Controls Cell Proliferation and Regulates the Proapoptotic Gene Hid in Drosophila. Cell, 113, 25-36. http://dx.doi.org/10.1016/S0092-8674(03)00231-9

[10] Liu, J., Xie, B., Chen, S., Jiang, F. and Meng, W. (2014) Association Study of Two Inflammation-Related Polymorphisms with Susceptibility to Hepatocellular Carcinoma: A Meta-Analysis. BMC Medical Genetics, 15, 92. http://dx.doi.org/10.1186/s12881-014-0092-7

[11] Rothschild, S.I., Tschan, M.P., Federzoni, E.A., Jaggi, R., Fey, M.F., Gugger, M. and Gautschi, O. (2012) MicroRNA29b Is Involved in the Src-ID1 Signaling Pathway and Is Dysregulated in Human Lung Adenocarcinoma. Oncogene, 31, 4221-4232. http://dx.doi.org/10.1038/onc.2011.578

[12] Calin, G.A., Ferracin, M., Cimmino, A., Di Leva, G., Shimizu, M., Wojcik, S.E., Iorio, M.V., et al. (2005) A MicroRNA Signature Associated with Prognosis and Progression in Chronic Lymphocytic Leukemia. The New England Journal of Medicine, 353, 1793-1801. http://dx.doi.org/10.1056/NEJMoa050995

[13] Lu, J., Getz, G., Miska, E.A., Alvarez-Saavedra, E., Lamb, J., Peck, D., Sweet-Cordero, A., et al. (2005) MicroRNA Expression Profiles Classify Human Cancers. Nature, 435, 834-838. http://dx.doi.org/10.1038/nature03702

[14] Li, J., Smyth, P., Flavin, R., Cahill, S., Denning, K., Aherne, S., Guenther, S.M., et al. (2007) Comparison of miRNA Expression Patterns Using Total RNA Extracted from Matched Samples of Formalin-Fixed Paraffin-Embedded (FFPE) Cells and Snap Frozen Cells. BMC Biotechnology, 7, 36. http://dx.doi.org/10.1186/1472-6750-7-36

[15] Schultz, J., Lorenz, P., Gross, G., Ibrahim, S. and Kunz, M. (2008) MicroRNA Let-7b Targets Important Cell Cycle Molecules in Malignant Melanoma Cells and Interferes with Anchorage-Independent Growth. Cell Research, 18, 549557. http://dx.doi.org/10.1038/cr.2008.45

[16] Chen, J., Feilotter, H.E., Pare, G.C., Zhang, X., Pemberton, J.G., Garady, C., Lai, D., et al. (2010) MicroRNA-193b Represses Cell Proliferation and Regulates Cyclin D1 in Melanoma. The American Journal of Pathology, 176, 2520 2529. http://dx.doi.org/10.2353/ajpath.2010.091061

[17] Caramuta, S., Egyhazi, S., Rodolfo, M., Witten, D., Hansson, J., Larsson, C. and Lui, W.O. (2010) MicroRNA Expression Profiles Associated with Mutational Status and Survival in Malignant Melanoma. Journal of Investigative Dermatology, 130, 2062-2070. http://dx.doi.org/10.1038/jid.2010.63

[18] Glud, M., Rossing, M., Hother, C., Holst, L., Hastrup, N., Nielsen, F.C., Gniadecki, R., et al. (2010) Downregulation 
of miR-125b in Metastatic Cutaneous Malignant Melanoma. Melanoma Research, 20, 479-484. http://dx.doi.org/10.1097/CMR.0b013e32833e32a1

[19] Philippidou, D., Schmitt, M., Moser, D., Margue, C., Nazarov, P.V., Muller, A., Vallar, L., et al. (2010) Signatures of MicroRNAs and Selected MicroRNA Target Genes in Human Melanoma. Cancer Research, 70, 4163-4173. http://dx.doi.org/10.1158/0008-5472.CAN-09-4512

[20] Yang, C. and Wei, W. (2011) The miRNA Expression Profile of the Uveal Melanoma. Science China Life Sciences, 54, 351-358. http://dx.doi.org/10.1007/s11427-011-4149-y

[21] Xu, Y., Brenn, T., Brown, E.R., Doherty, V. and Melton, D.W. (2012) Differential Expression of MicroRNAs during Melanoma Progression: MiR-200c, miR-205 and miR-211 Are Downregulated in Melanoma and Act as Tumour Suppressors. British Journal of Cancer, 106, 553-561. http://dx.doi.org/10.1038/bjc.2011.568

[22] Sand, M., Skrygan, M., Sand, D., Georgas, D., Gambichler, T., Hahn, S.A., Altmeyer, P., et al. (2013) Comparative Microarray Analysis of MicroRNA Expression Profiles in Primary Cutaneous Malignant Melanoma, Cutaneous Malignant Melanoma Metastases, and Benign Melanocytic Nevi. Cell and Tissue Research, 351, 85-98. http://dx.doi.org/10.1007/s00441-012-1514-5

[23] Segura, M.F., Belitskaya-Levy, I., Rose, A.E., Zakrzewski, J., Gaziel, A., Hanniford, D., Darvishian, F., et al. (2010) Melanoma MicroRNA Signature Predicts Post-Recurrence Survival. Clinical Cancer Research, 16, 1577-1586. http://dx.doi.org/10.1158/1078-0432.CCR-09-2721

[24] Poliseno, L., Haimovic, A., Segura, M.F., Hanniford, D., Christos, P.J., Darvishian, F., Wang, J., et al. (2012) Histology-Specific MicroRNA Alterations in Melanoma. Journal of Investigative Dermatology, 132, 1860-1868. http://dx.doi.org/10.1038/jid.2011.451

[25] Qi, M., Huang, X., Zhou, L. and Zhang, J. (2014) Identification of Differentially Expressed MicroRNAs in Metastatic Melanoma Using Next-Generation Sequencing Technology. International Journal of Molecular Medicine, 33, 11171121. http://dx.doi.org/10.3892/ijmm.2014.1668

[26] Zhang, X.Y., Liu, S.R., Hu, T.S., Liu, S.P., He, Y. and Sun, S.H. (2009) Up-Regulated MicroRNA-143 Transcribed by Nuclear Factor Kappa B Enhances Hepatocarcinoma Metastasis by Repressing Fibronectin Expression. Hepatology, 50, 490-499. http://dx.doi.org/10.1002/hep.23008

[27] Wang, J.L., Hu, Y., Kong, X., Wang, Z.H., Chen, H.Y., Xu, J. and Fang, J.Y. (2013) Candidate MicroRNA Biomarkers in Human Gastric Cancer: A Systematic Review and Validation Study. PLoS ONE, 8, e73683. http://dx.doi.org/10.1371/journal.pone.0073683

[28] Griffith, O.L., Melck, A., Jones, S.J. and Wiseman, S.M. (2006) Meta-Analysis and Meta-Review of Thyroid Cancer Gene Expression Profiling Studies Identifies Important Diagnostic Biomarkers. Journal of Clinical Oncology, 24, 5043-5051. http://dx.doi.org/10.1200/JCO.2006.06.7330

[29] Dennis Jr., G., Sherman, B.T., Hosack, D.A., Yang, J., Gao, W., Lane, H.C. and Lempicki, R.A. (2003) DAVID: Database for Annotation, Visualization, and Integrated Discovery. Genome Biology, 4, P3. http://dx.doi.org/10.1186/gb-2003-4-5-p3

[30] Weinstein, D., Leininger, J., Hamby, C. and Safai, B. (2014) Diagnostic and Prognostic Biomarkers in Melanoma. The Journal of Clinical and Aesthetic Dermatology, 7, 13-24.

[31] Meng, F., Henson, R., Wehbe-Janek, H., Ghoshal, K., Jacob, S.T. and Patel, T. (2007) MicroRNA-21 Regulates Expression of the PTEN Tumor Suppressor Gene in Human Hepatocellular Cancer. Gastroenterology, 133, 647-658. http://dx.doi.org/10.1053/j.gastro.2007.05.022

[32] Wickramasinghe, N.S., Manavalan, T.T., Dougherty, S.M., Riggs, K.A., Li, Y. and Klinge, C.M. (2009) Estradiol Downregulates miR-21 Expression and Increases miR-21 Target Gene Expression in MCF-7 Breast Cancer Cells. Nucleic Acids Research, 37, 2584-2595. http://dx.doi.org/10.1093/nar/gkp117

[33] Lu, T.X., Munitz, A. and Rothenberg, M.E. (2009) MicroRNA-21 Is Up-Regulated in Allergic Airway Inflammation and Regulates IL-12p35 Expression. The Journal of Immunology, 182, 4994-5002. http://dx.doi.org/10.4049/jimmunol.0803560

[34] Asangani, I.A., Rasheed, S.A., Nikolova, D.A., Leupold, J.H., Colburn, N.H., Post, S. and Allgayer, H. (2008) MicroRNA-21 (miR-21) Post-Transcriptionally Downregulates Tumor Suppressor Pdcd4 and Stimulates Invasion, Intravasation and Metastasis in Colorectal Cancer. Oncogene, 27, 2128-2136. http://dx.doi.org/10.1038/sj.onc.1210856

[35] Volinia, S., Calin, G.A., Liu, C.G., Ambs, S., Cimmino, A., Petrocca, F., Visone, R., et al. (2006) A MicroRNA Expression Signature of Human Solid Tumors Defines Cancer Gene Targets. Proceedings of the National Academy of Sciences of the United States of America, 103, 2257-2261. http://dx.doi.org/10.1073/pnas.0510565103

[36] Hossain, A., Kuo, M.T. and Saunders, G.F. (2006) Mir-17-5p Regulates Breast Cancer Cell Proliferation by Inhibiting Translation of AIB1 mRNA. Molecular and Cellular Biology, 26, 8191-8201. http://dx.doi.org/10.1128/MCB.00242-06 
[37] Kim, K., Chadalapaka, G., Lee, S.O., Yamada, D., Sastre-Garau, X., Defossez, P.A., Park, Y.Y., et al. (2012) Identification of Oncogenic MicroRNA-17-92/ZBTB4/Specificity Protein Axis in Breast Cancer. Oncogene, 31, 1034-1044. http://dx.doi.org/10.1038/onc.2011.296

[38] Jin, H.Y., Lai, M. and Xiao, C. (2014) MicroRNA-17 92 Is a Powerful Cancer Driver and a Therapeutic Target. Cell Cycle, 13, 495-496. http://dx.doi.org/10.4161/cc.27784

[39] Yu, Z., Willmarth, N.E., Zhou, J., Katiyar, S., Wang, M., Liu, Y., McCue, P.A., et al. (2010) MicroRNA 17/20 Inhibits Cellular Invasion and Tumor Metastasis in Breast Cancer by Heterotypic Signaling. Proceedings of the National Academy of Sciences of the United States of America, 107, 8231-8236. http://dx.doi.org/10.1073/pnas.1002080107

[40] Li, H., Gupta, S., Du, W.W. and Yang, B.B. (2014) MicroRNA-17 Inhibits Tumor Growth by Stimulating T-Cell Mediated Host Immune Response. Oncoscience, 1, 531-539. http://dx.doi.org/10.18632/oncoscience.69

[41] Kappelmann, M., Kuphal, S., Meister, G., Vardimon, L. and Bosserhoff, A.K. (2013) MicroRNA miR-125b Controls Melanoma Progression by Direct Regulation of c-Jun Protein Expression. Oncogene, 32, 2984-2991. http://dx.doi.org/10.1038/onc.2012.307

[42] Kozubek, J., Ma, Z., Fleming, E., Duggan, T., Wu, R., Shin, D.G. and Dadras, S.S. (2013) In-Depth Characterization of MicroRNA Transcriptome in Melanoma. PLoS ONE, 8, e72699. http://dx.doi.org/10.1371/journal.pone.0072699

[43] Liu, L.Y., Wang, J.N., Li, X.Q., Ma, J.H., Shi, C., Zhu, H.L., Xi, Q., et al. (2015) MiR-204-5p Suppresses Cell Proliferation by Inhibiting IGFBP5 in Papillary Thyroid Carcinoma. Biochemical and Biophysical Research Communications, 457, 621-626. http://dx.doi.org/10.1016/j.bbrc.2015.01.037

[44] Yin, Y., Zhang, B.B., Wang, W.L., Fei, B.J., Quan, C., Zhang, J.W., Song, M.X., et al. (2014) MiR-204-5p Inhibits Proliferation and Invasion and Enhances Chemotherapeutic Sensitivity of Colorectal Cancer Cells by Downregulating RAB22A. Clinical Cancer Research, 20, 6187-6199. http://dx.doi.org/10.1158/1078-0432.CCR-14-1030

[45] Finkel, T., Serrano, M. and Blasco, M.A. (2007) The Common Biology of Cancer and Ageing. Nature, 448, 767-774. http://dx.doi.org/10.1038/nature05985

[46] Berger, A.H., Knudson, A.G. and Pandolfi, P.P. (2011) A Continuum Model for Tumour Suppression. Nature, 476, 163-169. http://dx.doi.org/10.1038/nature10275 